\title{
A Simplified Analytical Solution of Mechanical Responses of Soil Subjected to Repeated Impact Loading
}

\author{
Futian Zhao, ${ }^{1,2,3}$ Jun Liu $\mathbb{D}^{1},{ }^{1,2,3}$ Zhimin Xiao, ${ }^{1,2,3}$ Mingqing Liu, ${ }^{1,2,3}$ Yue Wang, ${ }^{1,2,3}$ \\ Chen Ou, ${ }^{1,2,3}$ and Mengyang Zhen ${ }^{1,2,3}$ \\ ${ }^{1}$ College of Civil and Transportation Engineering, Hohai University, Nanjing 210098, China \\ ${ }^{2}$ Institute of Engineering Safety and Disaster Prevention, Hohai University, Nanjing 210098, China \\ ${ }^{3}$ Key Laboratory of Ministry of Education for Geomechanics and Embankment Engineering, Hohai University, \\ Nanjing 210098, China \\ Correspondence should be addressed to Jun Liu; ljun8@263.net
}

Received 26 May 2020; Revised 20 August 2020; Accepted 28 September 2020; Published 4 November 2020

Academic Editor: John D. Clayton

Copyright ( 92020 Futian Zhao et al. This is an open access article distributed under the Creative Commons Attribution License, which permits unrestricted use, distribution, and reproduction in any medium, provided the original work is properly cited.

\begin{abstract}
A simplified dynamic response model is proposed based on the deformation and dynamic stress response characteristics of soil under impact loading. The foundation is divided into two distinct zones: a projection cylinder acting vertically under impact loading and a hollow cylinder outside the projection area. It is assumed that the ramming deformation of the projected cylinder under the vertical impact load is a quasi-static loading process under the maximum contact dynamic stress through the quasistatic method, and the settlement calculation without lateral deformation is given. It is assumed that the inner wall of the hollow cylinder is subjected to horizontal lateral pressure and the analytical solution of the horizontal dynamic stress considering the plastic deformation of soil is given. The simplified dynamic response model can reflect the mechanical response of soil under impulse train load well which can provide reference for similar projects.
\end{abstract}

\section{Introduction}

Research studies on mechanical responses of soil subjected to impact loads have great prosperity in geotechnical engineering or other related fields. For instance, rocks falling off in a landslide impact the ground continuously, generating great influences on the mechanical properties of the soil. In demolition blasting, the vibration effect of the ground is induced by the continuous impact of the collapse body. In dynamic compaction, repeated impacts are used to improve the bearing capacity and stability of foundation. In a word, the mentioned situations are all related to the mechanical response of soils subjected to impact loading.

When the foundation is subjected to an impact load, different areas show different mechanical and deformation responses. Thilakasiri et al. [1] proposed the concept of impact zone according to the deformation characteristics of soil in 1996 and classified the impact area as three distinct zones: (1) a zone beneath the falling weight undergoing axial deformation while being in vertical motion, (2) an inner zone immediately surrounding the zone with shear deformation, and (3) an outer zone undergoing a relatively lower degree of shear deformation. In addition to the zoning characteristics of soil deformation, the distribution and attenuation $[2,3]$ of dynamic stress in the horizontal and vertical directions are also significantly different.

However, the focus of dynamic responses in different areas of soil foundation is different in practical projects. For the area directly affected by impact load (the area of vertical load projection), the focus is soil deformation. For example, dynamic consolidation $[4,5]$ technology was used to strengthen soft soil foundation. The technique uses the repeated dropping of heavy weight (tamper) on the weak ground that is going to be densified. For the outside of the projection area, the magnitude and distribution of dynamic stress were concerned. For example, in the demolition blasting [6] or dynamic compaction [7] in foundation treatment projects, the vibration of the construction site 
need to be monitored for evaluating the impact of shock wave on the surrounding structures [8-10].

Based on the concept of impact load affecting partition and engineering practice, a simplified dynamic response model is proposed in this paper. The foundation is divided into two distinct zones: a projection cylinder acting vertically under impact loading and a hollow cylinder outside the projection zone. The ramming deformation of the projected cylinder is assumed to be a quasi-static loading process under the maximum contact dynamic stress. Then, a settlement calculation method of the projected cylinder based on the effective stress principle is suggested. It is assumed that the inner wall of the hollow cylinder is subjected to the horizontal lateral pressure pulse. An analytical solution of the horizontal direction stress is obtained which takes the plastic deformation of soil into account.

\section{Simplified Dynamic Response Model of Soil under Impact Loading}

Based on the dynamic stress-strain responses, a simplified dynamic response model is proposed, and the following assumptions are made:

(1) The soil is continuous, isotropic, and homogeneous saturated clay.

(2) The soil is divided into two zones (as shown in Figure 1): One is the cylinder in which the impulse loading is shadowed. The other is the part outside the cylinder which can be considered as a hollow cylinder with infinite outer diameter.

(3) The cylinder was compressed without lateral deformation under the impulse loading.

(4) The inner wall of the hollow cylinder deforms in plane under horizontal lateral pressure. The lateral pressure is equal to the vertical pressure multiplied by the lateral stress coefficient. The material of the hollow cylinder is incompressible.

\section{Analytical Solution}

3.1. Load Calculation. The surface contact stress of impact load is the key factor to determine the strain and stress distribution. Qian et al. [11-15] have done research studies in theoretical analysis and experimental testing. They all found that although the time history curve of impact load have some fluctuations, there was only one sharp peak and no obvious second stress wave was observed. Therefore, the impact load was commonly simplified into one triangular wave or one half sine wave. Bai [13] pointed out that loading and unloading time of impact load had little influence on the total deformation of soil and the maximum contact dynamic stress governed the total strain. Therefore, the repeated impact load is simplified into the impulse train function in this paper (as shown in Figure 2), whose specific expression is

$$
P=\sum_{i=0}^{n} \sigma_{\max }^{i} \delta\left(t-t_{i}\right)
$$

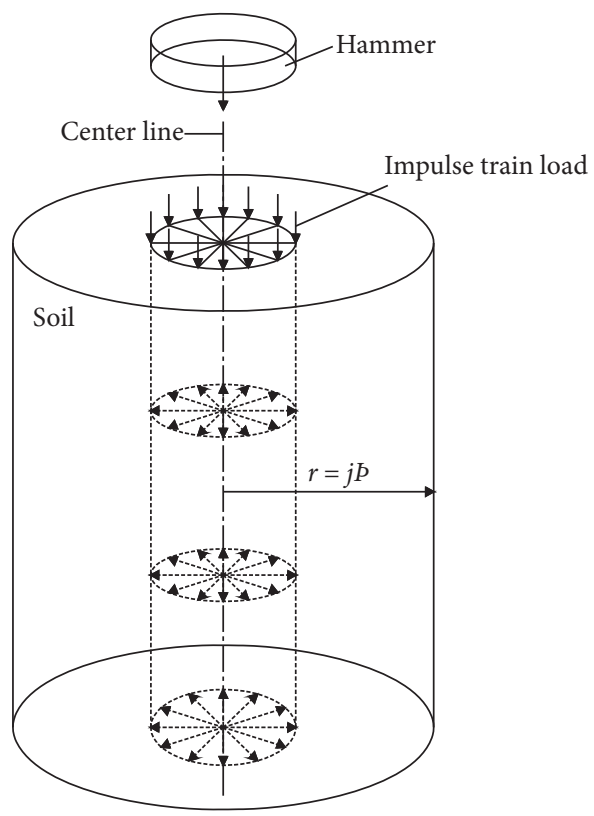

FIgURE 1: Schematic diagram of the simplified model of the soil under impulse loading.

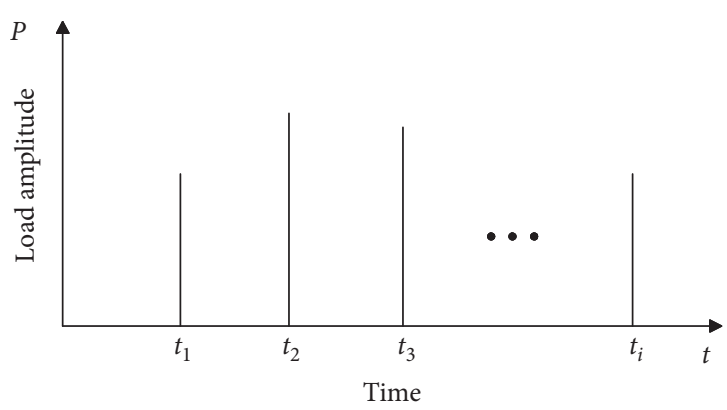

FIGURE 2: Schematic diagram of impulse loading function.

where $\sigma_{\max }^{i}$ is the maximum contact dynamic stress under the $i^{\text {th }}$ impulse load; $\sigma\left(t-t_{i}\right)$ is the impulse train function; and $t_{i}$ is the time of the $i^{\text {th }}$ impulse.

According to the structural dynamics theory, when solving the maximum contact dynamic stress, the influence of the cohesive force on the dynamic response can be ignored [16] and the maximum contact dynamic stress can be expressed as:

$$
\sigma_{\max }=\frac{u \sqrt{M S}}{\pi r^{2}}
$$

where $u$ is the speed of the hammer in contact with the ground; $S=\left(2 r E / 1-v^{2}\right)$ is the elastic constant; $v$ is Poisson's ratio; $E$ is the elastic modulus; and $M$ is the mass of the hammer.

3.2. Simplified Vertical Deformation Calculation of the Projection Cylinder. Bai $[13,14]$ proposed a method to calculate the consolidation deformation caused by dissipation of pore water pressure at the moment of dynamic compaction based 
on the systematic study on the distribution of pore water pressure and consolidation deformation. According to the quasi-static method, the calculation of the soil stress distribution under dynamic compaction $[1,11]$ can be simplified to static analysis with variable load. Therefore, the vertical dynamic stress distribution is similar to the internal stress distribution $\sigma_{z}$ of soil under static load [11], and the vertical dynamic stress can be expressed as

$$
\sigma_{z}=\sigma_{0}\left\{1-\frac{1}{\left[1+\left(r_{0} / z\right)^{2}\right]^{(3 / 2)}}\right\},
$$

where $\sigma_{0}$ is the contact dynamic stress; $r_{0}$ is the diameter of hammer; and $z$ is the calculation depth.

Based on Terzaghi infiltration consolidation theory, when the contact dynamic stress reaches the maximum, the pore water pressure [14] can be expressed as

$$
u_{z, t}=\frac{2 B \sigma_{\max }}{H} \cdot \sum_{n=1}^{\infty}\left[\int_{0}^{z} f(z) \sin \frac{N z}{H} \mathrm{~d} z\right] \sin \left(\frac{N z}{H}\right) e^{-N^{2}\left(C_{V} t / H^{2}\right),}
$$

where $B$ is the coefficient of Skempton pore pressure, considering that the pore water has certain compressibility under impact load, and the value of $B$ is taken as $0.95 ; C_{V}=$ $k(1+e) / a_{V} \gamma_{W}$ is the consolidation coefficient; $a_{V}$ is the compression coefficient; $k$ is the osmotic coefficient; $e$ is the void ratio; and $N=(2 n-1) \delta / 2$.

The effective stress increment caused by soil settlement can be expressed as

$$
\Delta p=\sigma_{z}-u_{z}
$$

The settlement $S_{t}$ is calculated with the equation of calculating compression without lateral deformation, which has the following form:

$$
S_{t}=\frac{a_{V}}{1+e_{0}} \Delta p H=\frac{a_{V}}{1+e_{0}} \int_{0}^{z} \Delta p \mathrm{~d} z
$$

3.3. Analytical Solution of Horizontal Dynamic Stress of the Hollow Cylinder. Figure 3 shows force analysis of hollow cylindrical soil under impact load. In Figure 3, $a$ is the inner diameter of the hollow cylinder and the radius of hammer; $b$ is the outer diameter of the hollow cylinder; $\sigma_{r}$ is the radial stress; $\sigma_{\theta}$ is the tangential stress; and $P_{i}$ is the lateral pressure acting on the inner wall of the hollow cylinder, which has the following form:

$$
P_{i}=\sum_{i=0}^{n} k_{i} \sigma_{\max }^{i} \delta\left(t-t_{i}\right)
$$

where $k_{i}$ is the lateral stress coefficient under the $i^{\text {th }}$ impact load.

The incompressibility of the hollow cylindrical material could be expressed as

$$
\frac{\partial U}{\partial r}+\frac{U}{r}=0
$$

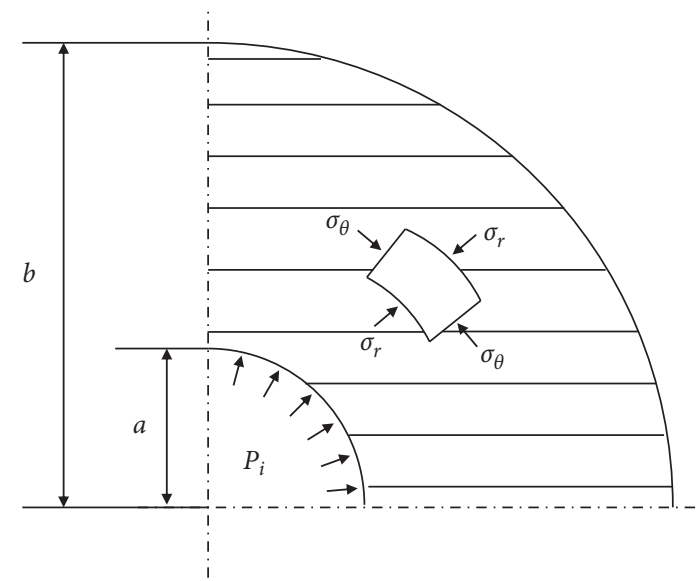

Figure 3: Schematic diagram of the hollow cylinder under plane stress.

where $U(r, t)$ is the radial displacement of a point in the cylinder.

By solving equation (9), we obtain

$$
U=\frac{C(t)}{r},
$$

and here, $C(t)$ is a function of time.

The differential motion equation has the following form:

$$
\frac{\partial \sigma_{r}}{\partial r}+\frac{\sigma_{r}-\sigma_{\theta}}{r}=\rho \frac{\partial^{2} U}{\partial t^{2}}
$$

where $\rho$ is the density of the material of the hollow cylinder.

In the case of plastic deformation, the following condition holds:

$$
\sigma_{r}-\sigma_{\theta}=-2 K
$$

where $K$ is the plastic constant; $K=\sigma_{T} / \sqrt{3}$ is the von Mises constant [17]; and $\sigma_{T}$ is the creep limit of the material.

Consequently, differential equation (10) reduces to the following form:

$$
\frac{\partial \sigma_{r}}{\partial r}=\rho \frac{\partial^{2} C}{\partial t} \cdot \frac{1}{r}+\frac{2 K}{r} .
$$

Integrating equation (12) and applying the boundary condition $r=a$ and $\sigma_{r}=P_{i}$ yield

$$
\sigma_{r}=-P_{i}+\left(2 K+\rho \frac{\partial^{2} C}{\partial t^{2}}\right) \ln \frac{r}{a},
$$

where $a$ is the internal radius of the hollow cylinder (Figure 3).

The equation for $\sigma_{r}$ in the elastic region has the following form [18]:

$$
\sigma_{r}=\rho \frac{\partial^{2} C}{\partial t^{2}} \ln \frac{r}{b}-2 \lambda\left(\frac{1}{r^{2}}-\frac{1}{b^{2}}\right) C(t),
$$

where $b$ is the external radius the hollow cylinder (Figure 3) and $\lambda$ is the Lame constant [19]. 
At the boundary between the elastic and plastic regions, the values determined by the expressions in (12) and (13) must coincide $\left(r=R^{2}(t)\right)$ :

$$
P_{i}-\left(2 K+\rho \frac{\partial^{2} C}{\partial t^{2}}\right) \ln \frac{R(t)}{a}=2 \lambda\left(\frac{1}{R^{2}(t)}-\frac{1}{b^{2}}\right) C(t)-\rho \frac{\partial^{2} C}{\partial t^{2}} \ln \frac{R(t)}{b} .
$$

Meanwhile, in the elastic region, there is [15]

$$
\sigma_{r}-\sigma_{\theta}=2 \lambda\left(\frac{\partial U}{\partial r}-\frac{U}{r}\right)=-4 \lambda \frac{C(t)}{r^{2}}
$$

and at the boundary, $r=R$, and equations (13) and (16) lead to

$$
\frac{4 \lambda C}{R^{2}}=2 K
$$

Consequently,

$$
C=\frac{K R^{2}}{2 \lambda}
$$

Applying $X(t)=R^{2}(t), A=(\rho / 2 \lambda) \ln (b / a)$, and equation (18) to equation (15) yields the equation of the plastic zone in the following form:

$$
A \frac{\partial^{2} X}{\partial t^{2}}+\ln X-\frac{X}{b^{2}}=\frac{P_{i}}{K}+\ln a^{2}-1 .
$$

The equation has the following initial conditions:

$$
t_{0}=T, \quad X=a^{2}, \quad \frac{\partial X}{\partial t}=\frac{2 \lambda}{K} \cdot \frac{\partial C}{\partial t},
$$

where $T$ is the moment at which the state of the material on the inner boundary of the cylinder is transferred to the plastic state. In addition, $T$ can be presented as the following form [18]:

$$
T=\frac{2}{\omega} \arcsin \sqrt{\frac{K\left(b^{2}-a^{2}\right)}{2 b^{2} P_{i}} .}
$$

The following requirement must be satisfied for the equation above:

$$
P_{i}>K \frac{\left(b^{2}-a^{2}\right)}{2 b^{2}}
$$

The quantity $\omega$ is determined by means of the following formula [18]:

$$
\omega=\frac{\sqrt{2 \lambda\left(b^{2}-a^{2}\right)}}{\rho a^{2} b^{2} \ln (b / a)}
$$

Similarly, the time function $C(t)$ can be determined by

$$
C(t)=\frac{a^{2} b^{2} P_{i}}{2 \lambda\left(b^{2}-a^{2}\right)}(1-\cos \omega t) .
$$

Equation (13) for determining the values of $\sigma_{r}$ in the plastic region is transformed by substituting equation (18) into it, resulting in the form

$$
\sigma_{r}=\frac{\ln (r / a)}{\ln (b / a)}\left(P_{i}+K\left(\ln \frac{b^{2}}{X}+\frac{X}{b^{2}}-1\right)\right)-P_{i} .
$$

By condition (10), $\sigma_{\theta}$ can be consequently determined by

$$
\sigma_{r}=\frac{\ln (r / a)}{\ln (b / a)}\left(P_{i}+K\left(\ln \frac{b^{2}}{X}+\frac{X}{b^{2}}-1\right)\right)-P_{i}+2 K \text {. }
$$

\section{Experimental Verification}

4.1. Test Setup. A type of self-developed testing equipment was designed to verify the simplified dynamic response model. As shown in Figure 4, the test equipment consisted of a trip, a drop hammer, and a wooden model box. Figure 4 is reproduced from the study by Xie et al., 2016 (under the Creative Commons Attribution License/public domain).

The model box was made of wooden plates with a thickness of $2 \mathrm{~cm}$, the height of the box was $120 \mathrm{~cm}$, and the horizontal dimensions were $80 \mathrm{~cm}$ by $80 \mathrm{~cm}$. The box was strengthened by nailing steel angles at four corners to prevent failure caused by soil pressure.

Clay was dried, crushed, sieved, and filled in layers in the model box. The soil properties were obtained by the triaxial test, as shown in Table 1.

The tripod and hammer were used to apply impact load. The drop hammer is a cylinder with a diameter of $135 \mathrm{~mm}$, a height of $14.5 \mathrm{~mm}$, and a weight of $16.8 \mathrm{~kg}$. The impact loads were applied by raising the drop hammer to the height of $0.8 \mathrm{~m}$ and let it fall off freely.

Tiny earth pressure gauges were placed in the designated location to measure the stress distribution. The dynamic stress decreased along the soil depth, so the spacing of earth pressure gauges at the lower part of the model box was smaller than that at the upper part. In order to measure the horizontal stress distribution, the earth pressure gauges were arranged symmetrically within a certain distance from the center line of the hammer in the same plane. Figure 5 shows the layout of earth pressure gauges in the vertical and horizontal directions.

\subsection{Test Procedures}

(1) A $10 \mathrm{~cm}$ thick layer of dry sand was placed at the bottom of the model box and covered with a layer of geotextile to prevent the mixture of sand and clay.

(2) The soil samples were filled to the model box layer by layer. Meanwhile, the earth pressure gauges were installed to measure the stress. The clear distance between the earth pressure gauges was maintained 4 times the diameter of the earth pressure gauges to minimize the interruptions.

(3) When soil was filled to the specified depth, the tripod and hammer were set to the designated location to apply impact loads. 

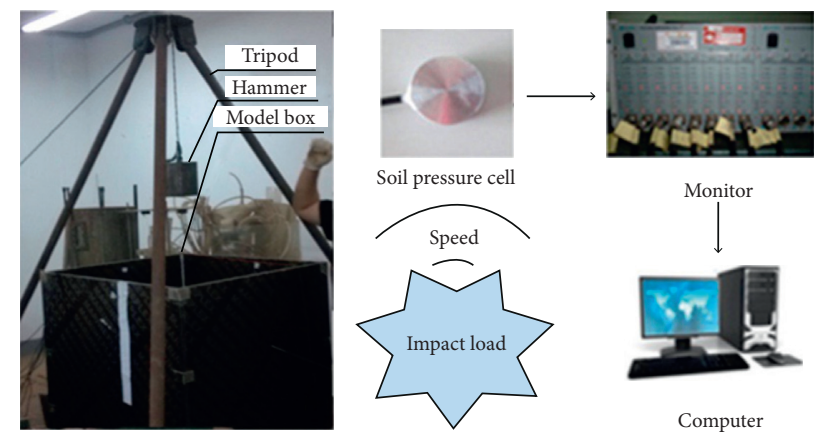

FIGURE 4: Experimental apparatus: (a) setup; (b) instrumentation.

TABLE 1: Soil properties.

\begin{tabular}{lccccc}
\hline$\omega(\%)$ & $\rho\left(\mathrm{g} / \mathrm{cm}^{3}\right)$ & $e$ & $E_{s}(\mathrm{MPa})$ & $c(\mathrm{kPa})$ & $\varphi\left({ }^{\circ}\right)$ \\
\hline 24.6 & 1.89 & 0.876 & 5.03 & 8.0 & 12.5 \\
\hline
\end{tabular}

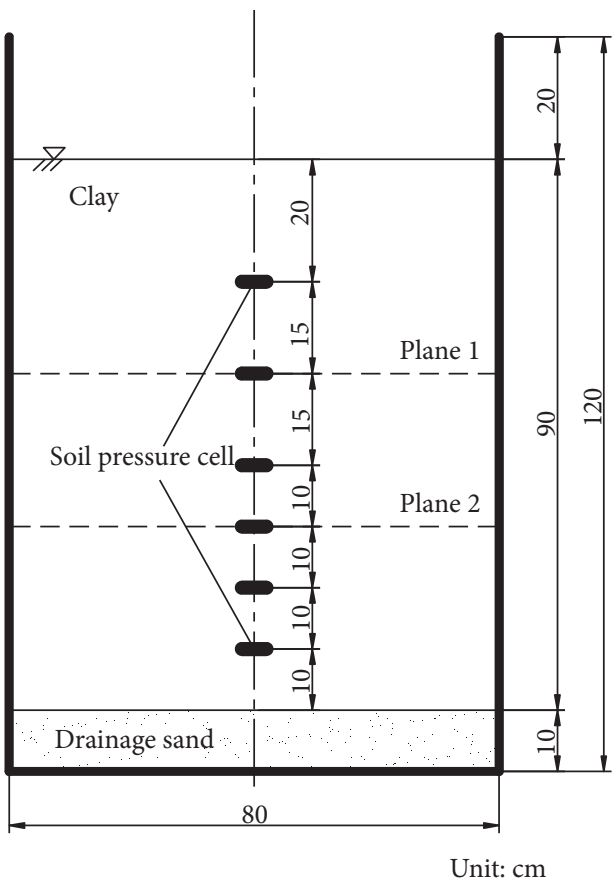

(a)

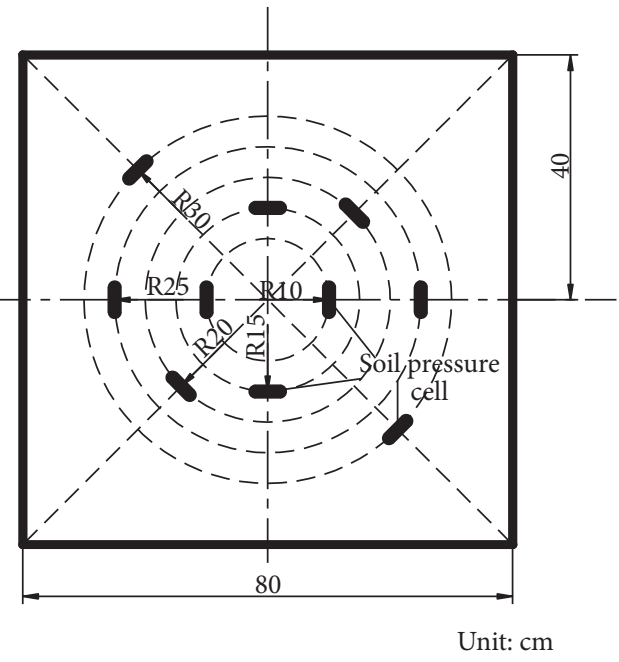

(b)

FIGURE 5: Layout of earth pressure boxes: (a) in the vertical direction; (b) in the horizontal direction.

\subsection{Experiment Result Analysis and Model Validation}

4.3.1. Deformation of the Projected Cylinder. Figure 6 shows the deformation process of the ramming hole with the distance of $0.8 \mathrm{~m}$ between adjacent hammer falling locations. The shapes of cylindrical holes were close to that of the drop hammer. The depths of cylindrical holes increased exponentially with the ramming times, and the diameters of ramming holes were not affected by the number of ramming times. When the ramming times were larger than 10 , the deformation of ramming holes tended to be stable, forming a cylindrical ramming hole with a depth of about $100 \mathrm{~mm}$ and a diameter of about $150 \mathrm{~mm}$. The experiment results show that it was reasonable to assume that the soil under the impact load of the drop hammer was a cylinder in the horizontal projection of the surface under impulse load.

4.3.2. Settlement Calculation of the Projected Cylinder. When the first ramming with a distance of $0.8 \mathrm{~m}$ between adjacent hammer falling locations was applied, the maximum dynamic stress on the contact surface between the 


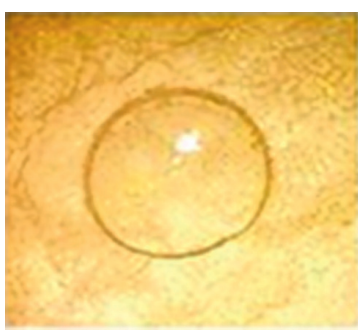

(a)

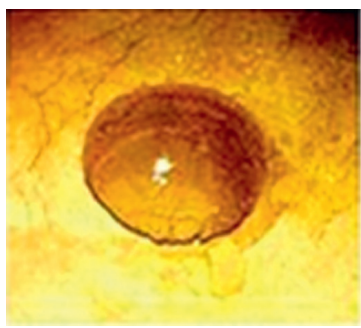

(d)

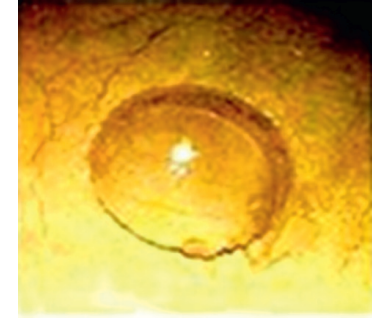

(b)

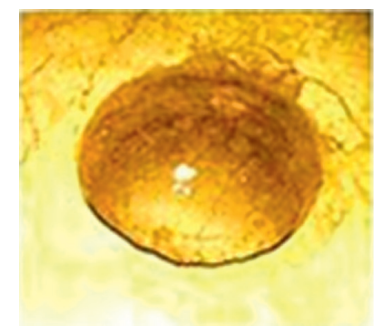

(e)

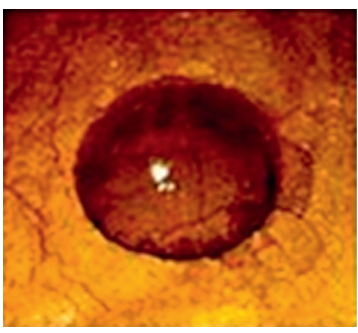

(c)

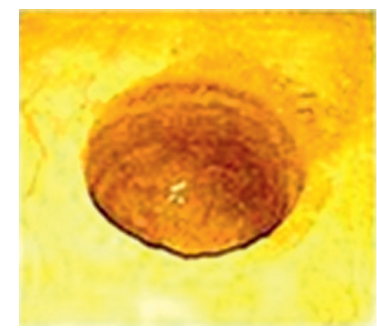

(f)

Figure 6: Deformation of impacted holes with the drop height of $0.8 \mathrm{~m}$.

drop hammer and soil can be calculated with equation (1) as $0.28 \mathrm{MPa}$. During the ramming process, the ramming depth was recorded to calculate the next maximum dynamic stress on the contact surface. The thickness of soil layer was chosen as $z=0.9 \mathrm{~m}$; Poisson's ratio was selected as $v=0.3$; and the elastic modulus was selected as $E=5 \mathrm{MPa}$. The change of Poisson's ratio and elastic modulus were ignored during compaction. Before the first compaction, the compressibility and the coefficient of consolidation were $a_{V}=1.2 \times 10^{-6}\left(\mathrm{~m}^{2} / \mathrm{N}\right)$ and $C_{V}=0.002\left(\mathrm{~m}^{2} / \mathrm{s}\right)$, and the other parameters are listed in Table 1. Equations (2) and (3) are used to calculate the distribution of internal stress and pore water pressure with depth when the contact dynamic stress reaches the maximum value. The calculated results are substituted into equations (4) and (5) for integral calculation to obtain the settlement.

The ramming process is considered as a quasi-static loading process under the maximum dynamic contact stress in the simplified settlement calculation. However, the change of soil properties under continuous impact load cannot be ignored, and especially, the reduction of void ratio will lead to the nonlinear attenuation of compression coefficient and consolidation coefficient. Since the attenuation of compression coefficient and consolidation coefficient is caused by the reduction of void ratio [17], an attenuation coefficient $\eta$ is proposed to describe the change of settlement characteristics in the compaction process. The variation of void ratio with the number of compaction was recorded (as shown in Figure 7) to describe the process quantitatively. It can be seen in Figure 7 that the void ratio exhibits nonlinear attenuation with ramming times. The relationship between the pore ratio and ramming times is determined with curve fitting to satisfy exponential attenuation, and the equation of $\eta$ can be established as

$$
\eta=e^{(-x / A)}
$$

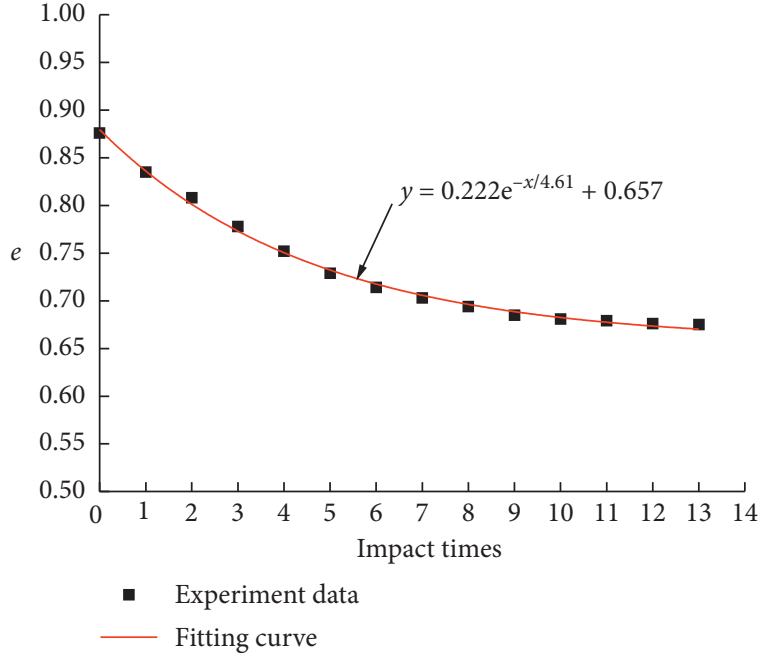

Figure 7: Relationship between void ratio and the number of ramming times.

where $x$ is the ramming times and $A$ is the constant related to the pore ratio change, and it is used to describe the attenuation degree of void ratio. According to the attenuation fitting function of void ratio, the value of $A$ is determined to be 4.61 .

Based on the established attenuation coefficient equation, the calculated values of compression coefficient and consolidation coefficient of projected soil after a certain number of ramming were determined and the settlement can be obtained. Figure 8 shows the comparison curve of measured and calculated compaction values of projected soil. It can be seen that the calculated values and measured values are in good agreement within the first 6 ramming. With the increase of the number of ramming times, the calculated value becomes smaller than the test values 


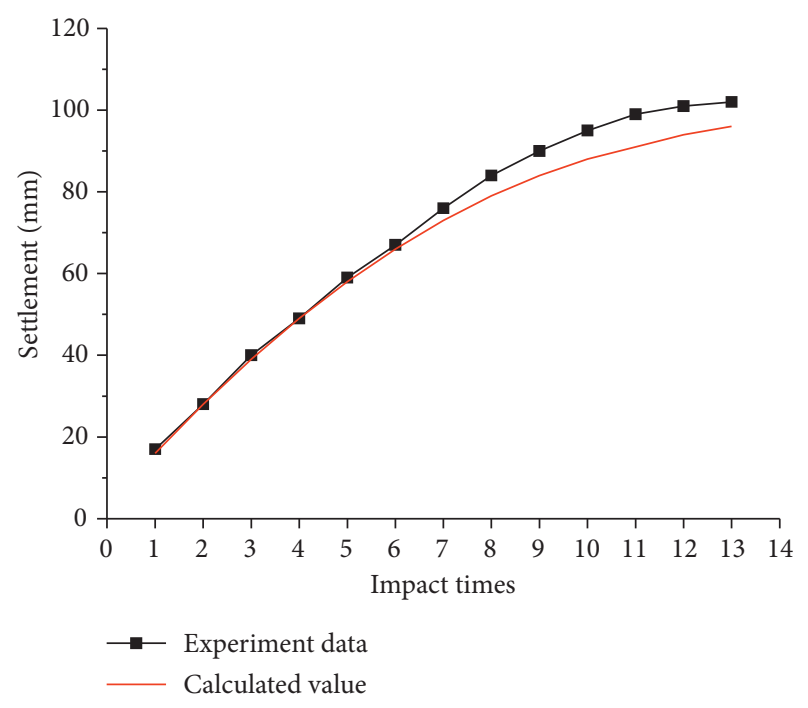

FIGURE 8: Relationship between soil settlement and the number of ramming times.

gradually. The main reason is that the deformation of the projected cylinder is considered as an ideal completely confined compression in the theoretical calculation, while the settlement caused by lateral deformation in the actual ramming process is ignored [20].

4.3.3. Dynamic Response Analysis of Hollow Cylindrical Soil. The value of lateral stress coefficient $k$ plays a decisive role in the dynamic response of hollow cylindrical soil and $k$ is closely related to the stress history. It is assumed that the initial lateral stress coefficient $k_{0}$ is the static lateral pressure coefficient, and it is defined as

$$
k_{0}=K_{0}=1-\sin \varphi^{\prime} .
$$

The dynamic consolidation process of the projected cylinder can be interpreted as the reduction of void ratio. The void ratio decreases with the increase in the number of ramming times, and the void ratio variation $\Delta e$ is positively correlated with the maximum dynamic stress and ramming times [21], while the internal friction angle $\varphi$ is negatively correlated with the void ratio $e$ [22]. It can be seen that the lateral stress coefficient attenuates to some extent in the ramming process. Similarly, the attenuation coefficient defined above is used to describe the attenuation degree of the lateral stress coefficient. Therefore, the lateral stress coefficient $k$ can be expressed as

$$
k=\eta K_{0}=e^{(-x / A)} K_{0} .
$$

With the governing equation of plastic zone radius equation (19), the plastic zone radius is determined. Here, $a=0.0675 \mathrm{~m}$ (hammer radius), $b$ is an infinite boundary, $K=0.1 \mathrm{MPa}$ (von Mises constant), and $\sigma_{T}$ is the creep limit $\left(\sigma_{T}=0.2 \mathrm{MPa}\right)$. Figure 9 shows the change of plastic zone radius. With the increase of ramming times, the plastic zone radius gradually increases to $0.136 \mathrm{~m}$ and then becomes stable. Therefore, the plastic

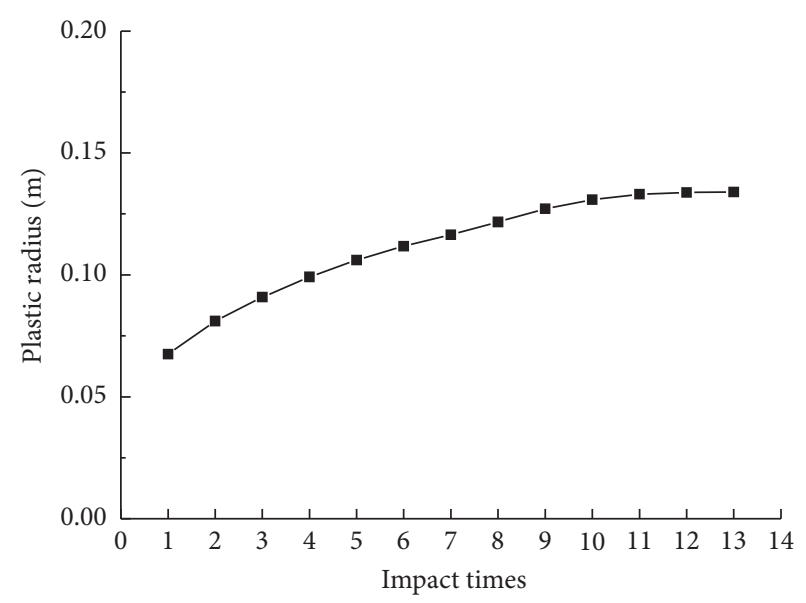

Figure 9: Relationship between plastic radius and the number of impact times.

zone radius is about 2 times the hammer radius, and the result is taken as the effective reinforcement range of hollow cylindrical soil [23].

Figure 10 shows the comparison of horizontal stress test results and analytical solution after the first compaction. It can be seen that the soil stress decays approximately as a logarithmic function along the horizontal radial direction, and the attenuation rate gradually decreases with the increase of the horizontal distance. The experiment results are in good agreement with the theoretical values.

4.4. Parameter Analysis. The relationships of the settlement to the number of impact loadings for different drop heights are shown in Figure 11. The settlement of the first impact was always the largest, and with the increase of the number of impacts, the soil settlement increases nonlinearly and tends to be stable. Although the settlement due to every single impact was unequal for each set of tests under a given impact energy, the eventual total settlement of each set of tests was nearly the same, converging to a critical value. It shows that the drop height has little influence on the final settlement but has great influence on the settlement development pattern.

Figure 12 shows the distribution of horizontal radial stress under different drop heights. It can be seen that the greater the impact height, the greater the corresponding stress value. But, the attenuation of the radial stress in the horizontal direction under different heights is close to each other.

When the impact energy is the same, the distribution of horizontal radial stress with different lateral stress coefficient values is given in Figure 13. The results show that the lateral stress coefficient not only has great influence on the value of dynamic stress at each point but also changes the attenuation law of stress. The attenuation of radial stress in the horizontal direction becomes more obvious when the value of the lateral stress coefficient is small. 


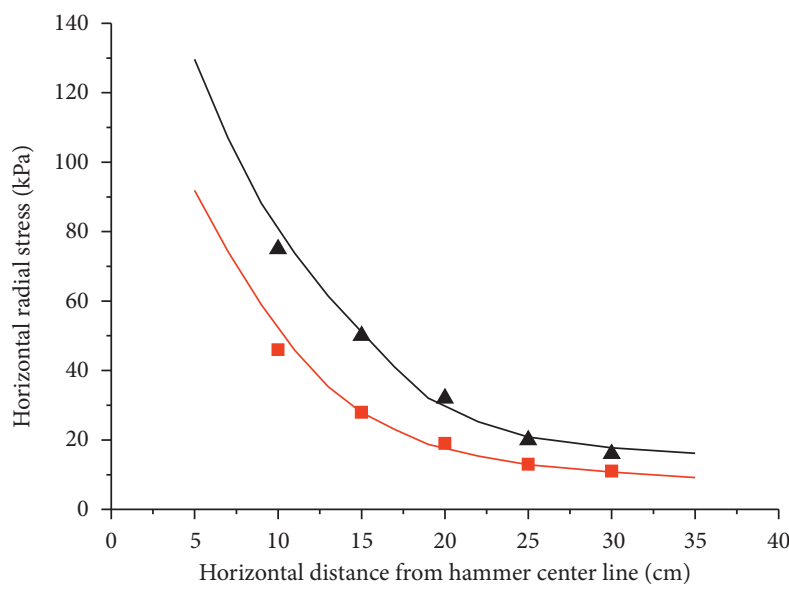
- Experiment data-plane 1
- Experiment data-plane 2
Calculated value-plane 1 Calculated value-plane 2

Figure 10: Comparison of analytic solutions and test results of horizontal stress after the first compaction.

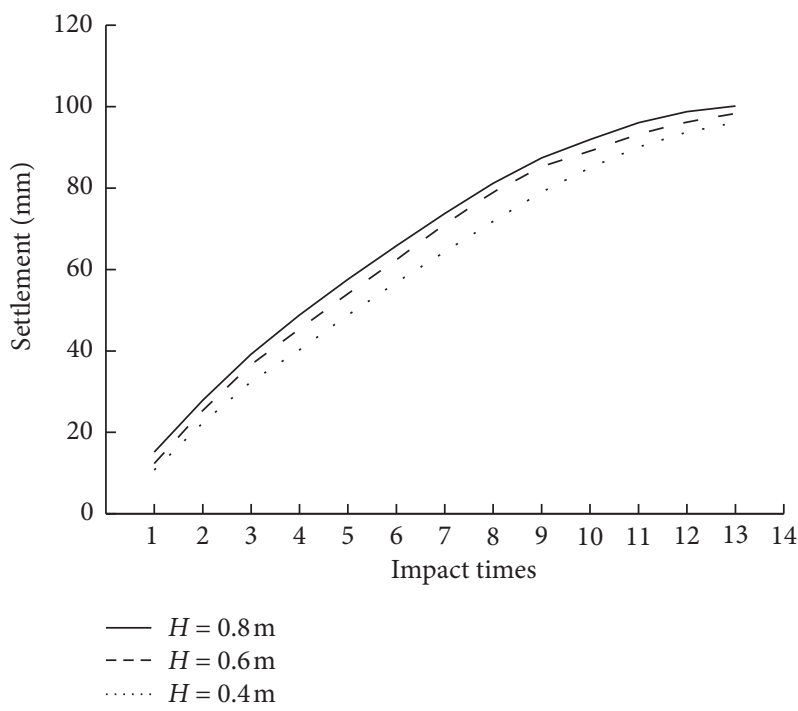

FIGURE 11: Relationship between soil settlement and the number of ramming times under different drop heights.

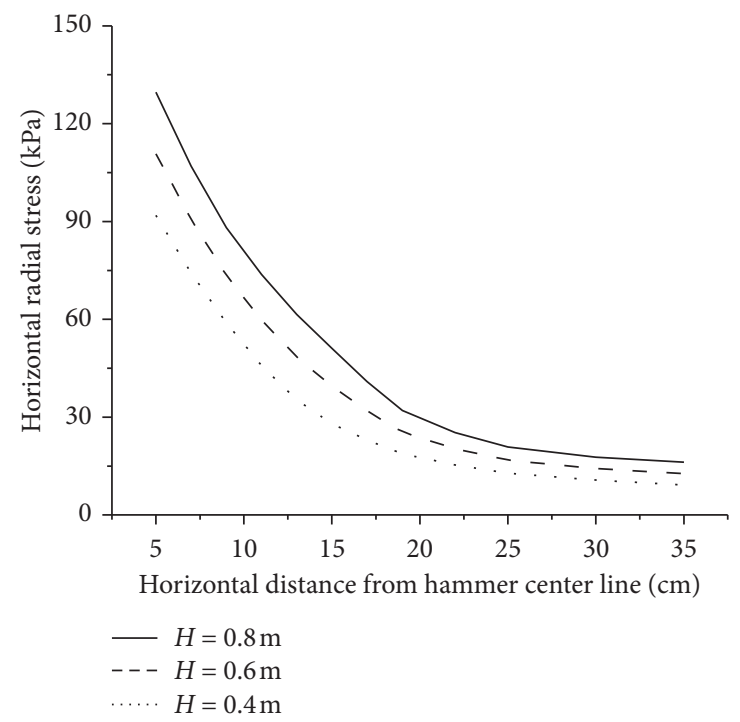

FIgURE 12: The distribution of horizontal radial stress under different drop heights. 


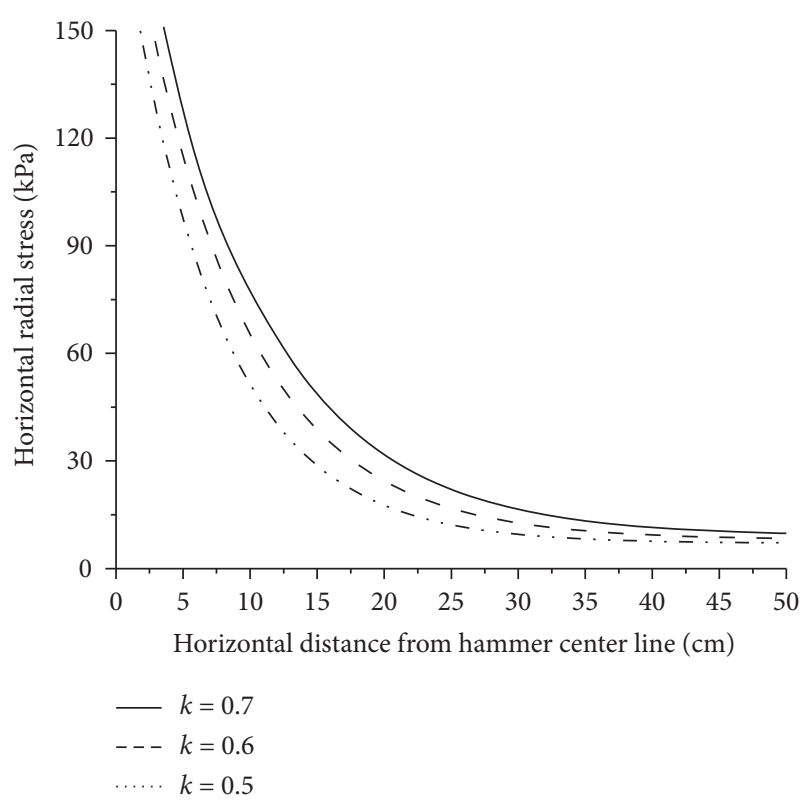

FIgURE 13: The distribution of horizontal radial stress with different lateral stress coefficient values.

\section{Conclusions}

(1) The simplified dynamic response model can well predict the mechanical response of soil under impulse train load.

(2) The changes of physical and mechanical properties of projective cylindrical soil under continuous impact load cannot be ignored. The attenuation coefficient, $\eta$, was proposed to describe the variation degree of settlement characteristic and dynamic stress response of soil during impulse train load. The calculation equation is $\eta=e^{(-x / A)}$, where $x$ is the ramming times; $A$ is the constant related to the change of the pore ratio; and $e$ is used to describe the attenuation degree of the void ratio.

(3) The governing equation of the horizontal plastic zone radius was established to calculate the plastic zone radius of hollow cylindrical soil. The calculated value of plastic zone radius was about 2 times of the hammer radius, and the solution was taken as the effective consolidation range of hollow cylindrical soil. The lateral stress coefficient is the main consideration to determine the radius of the plastic zone.

\section{Data Availability}

The data used to support the findings of this study are available from the corresponding author upon request.

\section{Conflicts of Interest}

The authors declare that they have no conflicts of interest regarding the work reported in this paper.

\section{Authors' Contributions}

Futian Zhao analyzed data and wrote the paper. Jun Liu contributed data. Zhimin Xiao, Mingqing Liu, Yue Wang, Chen Ou, and Mengyang Zhen involved in experimental testing.

\section{Acknowledgments}

This work was supported by the National Natural Science Foundation of China (nos. 51874118 and 51778211), the Fundamental Research Funds for the Central Universities (no. KYCX18-0567), and the Jiangsu Provincial Natural Science Foundation, China (no.BK20171434).

\section{References}

[1] H. S. Thilakasiri, M. Gunaratne, G. Mullins, P. Stinnette, and B. Jory, "Investigation of impact stresses induced in laboratory dynamic compaction of soft soils," International Journal for Numerical and Analytical Methods in Geomechanics, vol. 20, no. 10, pp. 753-767, 1996.

[2] M. Lysmer and F. E. Richart, "Dynamic response of footing to vertical loading," Journal of the Soil Mechanics and Foundations Division, vol. 92, no. 1, pp. 65-91, 1966.

[3] P. W. Mayne and J. S. Jones, "Impact stresses during dynamic compaction," Journal of Geotechnical Engineering, vol. 109, no. 10, pp. 1342-1346, 1983.

[4] L. Ménard and Y. Broise, "Theoretical and practical aspect of dynamic consolidation," Géotechnique, vol. 25, no. 1, pp. 3-18, 1975.

[5] Y. K. Chow, D. M. Yong, K. Y. Yong, and S. L. Lee, "Dynamic compaction analysis," Journal of Geotechnical Engineering, vol. 118, no. 8, pp. 1141-1157, 1992.

[6] S. Ji, W. P. Xie, and L. Wang, "Tests for ground vibration characteristics and propagation laws due to blasting and touchdown impact," Chinese Journal of Vibration and Shock, vol. 37, no. 11, pp. 195-201, 2018.

[7] J. H. Hwang and T. Y. Tu, "Ground vibration due to dynamic compaction," Soil Dynamics and Earthquake Engineering, vol. 26, no. 5, pp. 337-346, 2006.

[8] X. Q. Fang, T. F. Zhang, B. L. Li, and R. J. Yuan, "Elastic-slip interface effect on dynamic stress around twin tunnels in soil medium subjected to blast waves," Computers and Geotechnics, vol. 119, Article ID 103301, 2020.

[9] C.-S. Zhu, X.-Q. Fang, J.-X. Liu, and H.-Y. Li, “Surface energy effect on nonlinear free vibration behavior of orthotropic piezoelectric cylindrical nano-shells," European Journal of Mechanics-A/Solids, vol. 66, pp. 423-432, 2017.

[10] B. L. Li, X. Q. Fang, T. F. Zhang, and S. P. Yang, "Elastic-slip interface effect on dynamic response of underwater convey tunnel in saturated poroelastic soil subjected to plane waves," Tunnelling and Underground Space Technology, vol. 103, Article ID 103468, 2020.

[11] J. Qian, X. Qian, W. Zhao, and F. Shuai, "Theory and practice of dynamic consolidation," Chinese Journal of Geotechnical Engineering, vol. 8, no. 6, pp. 1-17, 1986.

[12] C. M. He, J. F. Zou, and L. Li, "Field tests on measurement of dynamic stress of dynamic compaction," Chinese Journal of Geotechnical Engineering, vol. 29, no. 4, pp. 628-632, 2007.

[13] B. Bai, "Calculation of consolidation deformation of saturated stratum under dynamic compaction loading," Chinese Journal of Rock and Soil Mechanics, vol. 24, no. 1, pp. 57-60, 2003. 
[14] B. Bai, "Pore water pressure of saturated stratum under dynamic impacts," Chinese Journal of Rock Mechanics and Engineering, vol. 22, no. 9, pp. 1469-1473, 2003.

[15] Z. R. Niu and G. T. Yang, "Studies on the displacement of soils subjected to the impact loading," Chinese Journal of Rock and Soil Mechanics, vol. 26, no. 11, pp. 1743-1748, 2005.

[16] H. L. Liu, Y. B. Gao, J. J. Cao, and S. L. Xu, "Calculation of contact stress and soil vertical displacement under dynamic compaction," Chinese Journal of Geotechnical Engineering, vol. 31, no. 10, pp. 1493-1497, 2009.

[17] H. G. Hopkins, "Dynamic inelastic deformations of metals," Applied Mechanics Reviews, pp. 417-431, 1961.

[18] R. P. Didyk and E. A. Masakovskii, "Investigation of field of dynamic stresses with impulse loading," International Applied Mechanics, vol. 35, no. 2, pp. 170-174, 1999.

[19] J. D. Achenbach, Wave Propagation in Elastic Solids, NorthHolland, Amsterdam, Netherlands, 1980.

[20] H. Y. Xu, Z. F. Zhou, and Z. Q. Gao, "Experimental study on coefficient of land subsidence," Chinese Journal of Geotechnical Engineering, vol. 33, no. 12, pp. 1969-1973, 2011.

[21] K. F. Mostafa and R. Y. Liang, "Numerical modeling of dynamic compaction in cohesive soils," Geo-frontiers Congress, 2011.

[22] K. Shinohara, M. Oida, and B. Golman, "Effect of particle shape on angle of internal friction by triaxial compression test," Powder Technology, vol. 107, no. 1-2, pp. 131-136, 2000.

[23] X. Xie, Y. Yao, J. Liu, P. Li, and G. Yang, "Mechanical behavior of unsaturated soils subjected to impact loading," Shock and Vibration, vol. 2016, no. 1, Article ID 4703981, 2016. 